\title{
Channel Model and Throughput Analysis for LEO OFDM Satellite Communication System
}

\author{
Zhenyu $\mathrm{Na}^{1}$, Qinyang $\mathrm{Guan}^{2}, \mathrm{Ce} \mathrm{Fu}^{1}$, Yang $\mathrm{Cui}^{3}$ and Qing Guo ${ }^{2}$ \\ ${ }^{1}$ School of Information Science and Technology, Dalian Maritime University \\ ${ }^{2}$ Communication Research Center, Harbin Institute of Technology \\ ${ }^{3}$ Institute of Science and Industry Technology, Harbin Institute of Technology \\ ${ }^{1}$ nazhenyu@dlmu.edu.cn,fu_ce@dlmu.edu.cn, ${ }^{2}$ mingyun_hit@163.com, \\ ycui@hit.edu.cn
}

\begin{abstract}
Low earth orbit (LEO) satellite has the advantages of global coverage, low propagation delay and low path losses, etc. Combined with orthogonal frequency division multiplexing (OFDM) transmission, LEO satellite system can support very high capacity suitable for wideband multimedia traffic. This paper analyzes the channel model and throughput for LEO OFDM satellite communication system. First, several channel models for LEO satellite system are expounded with simulations because channel model is the basis of throughput analysis. Second, the beam model and the signaling model for LEO satellite are introduced. Considering high speed movement of LEO satellite, channel fading and the channel quality information (CQI), link throughput is simulated. Finally, compared with MF-TDMA system, simulation results demonstrate that OFDM system outperforms the former because its feasibility and superiority in throughput.
\end{abstract}

Keywords: LEO satellite, OFDM, Channel model, Throughput analysis, MF-TDMA

\section{Introduction}

The future generation of communication system will be featured by their wireless mobility, global coverage and broadband high-speed transmission. Considering the tendency, satellite communication is an effective alternative of global communications. As satellite communication is not subject to terrains and oceans, it plays an irreplaceable role in future communication networks. Besides, compared with geosynchronous earth orbit (GEO) satellite, LEO satellite has the advantages of polar coverage, flexible networking, low propagation loss and delay, supporting airborne devices and diverse handsets to directly communicate with satellites that have been widely deployed in many commercial systems [1].

However, as LEO satellite communication suffers from frequency selective fading and limited spectrum, an efficient and well-performed transmission scheme should be adopted to get over these disadvantages. OFDM using orthogonal subcarriers, not only reinforces the ability to resist frequency selective fading, but has higher spectrum utilization. In addition, OFDM supports flexible subcarrier allocation which is suitable for the asymmetrical data rate on both uplink and downlink of satellite communication system. Therefore, OFDM applied to satellite system is significant and promising.

High-speed data transmission is dependent on high throughput. In order to increase throughput of LEO OFDM satellite system, adaptive modulation and coding (AMC) can be used. AMC adapts to channel fading according to maximizing throughput and improving 
spectrum efficiency. In this paper, to analyze throughput of LEO OFDM satellite system, link model covered by satellite beam should be established. Throughput is relevant to many factors, including satellite trajectory, movement speed and system bandwidth. Especially, satellite channel is so time-varying and dynamic that system throughput is highly affected by channel characteristics. Therefore, channel modeling for LEO satellite system will be analyzed firstly. In present, throughput analysis for terrestrial wireless OFDM systems is expounded in literature [2-4]. This paper establishes AMC link model for LEO OFDM satellite system based on which its throughput analysis is presented.

\section{Flow of Throughput Analysis}

This section establishes the link model of LEO OFDM satellite communication system, which is the basis of analysis of beam coverage and throughput. It is should be considered that a number of data frames are simulated when user terminal (UT) moves in satellite beam coverage. The flowchart of throughput analysis for LEO OFDM satellite system is presented in Figure 1.

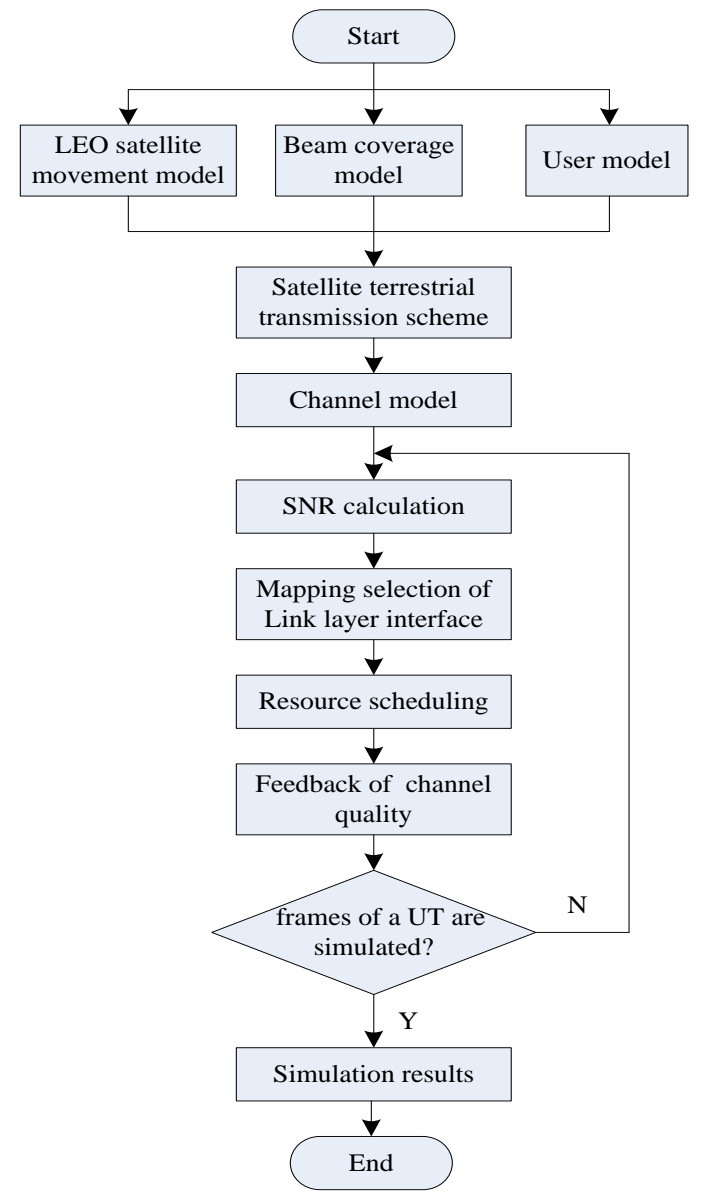

Figure 1. Flowchart of Throughput Analysis

For LEO satellite link, beam coverage model should be established. Satellite antenna produces spot beams which can be classified into fixed spot beam coverage, non-fixed spot beam coverage and shaped beam coverage. For LEO satellite system, non-fixed beam 
coverage is adopted more often than not. For LEO satellite system with multiple antennas, the coverage of one satellite consists of multiple spot beams as shown in Figure 2. Since beam coverage is relatively unchanged, the beam coverage model for LEO satellite can be simplified that the it can be divided into several hexagon cells. $R$ is the radius of inscribed circle of spot beam of which value ranges from $R_{\min }$ to $R_{\max }$ according to UT's position relative to LEO satellite. Specifically, if the elevation angle of satellite reaches the maximum, $R=R_{\min }$; otherwise, $R=R_{\max }$. For simplicity, the user model covered by satellite spot beam is approximated as hexagon and the radius of the beam is set $R=0.5\left(R_{\min }+R_{\max }\right)$.

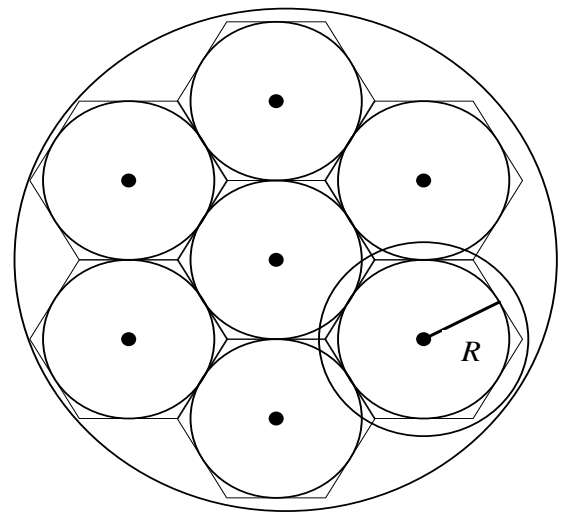

\section{Figure 2. Hexagon Model for LEO Satellite Beam Coverage}

For LEO OFDM satellite communication system, different signaling should be adopted on both uplink and downlink. Such signaling is transmitted in specific control channels. Specifically, UT sends satellite a connection request signal which is used to measure CQI of uplink, allocate and schedule resource to user. LEO satellite calculates the priority of user request so that the vacant resource can be allocated the user with high priority. The CQI of downlink can be acquired according to downlink transmitted signal, and then it is sent back to satellite. Therefore, satellite achieves AMC in the light of the CQI. In the other hand, CQI is determined by all subcarriers' SNR. When the requirement for bit error rate (BER) is strict, the hybrid automatic repeat request (HARQ) scheme is introduced to resend the erroneous frames.

\section{Channel Model of LEO Satellite Communication System}

In LEO satellite communications, the bandwidth of high-speed transmitted signal is more than the coherence bandwidth of the channel, leading to frequency selective fading of channel. In general, the wide-sense stationary un-correlative scattering (WSSUS) model is used to model frequency selective fading channel. Such channel is often described as the cascade of time-varying impulse response linear filter in which the altitude superposition of multi-paths and the delay of multi-paths are the parameters of the filter.

Let $h(t, \tau)$ denote the impulse response of frequency selective fading channel at time $t-\tau$, which can be expressed as

$$
h(t, \tau)=\sum_{n=0}^{L-1} A_{n}(t) \exp \left[-j \theta_{n}(t)\right] \delta\left(\tau-\tau_{n}(t)\right)
$$


where, $L$ is the number of multi-paths, $\left\{A_{n}(t)\right\}_{n=1}^{L}$ is the amplitude of the channel, $\left\{\theta_{n}(t)\right\}_{n=1}^{L}$ is the stochastic phase of the channel, $\left\{\tau_{n}(t)\right\}_{n=1}^{L}$ represents the delay of different paths.

LEO satellite is the supplement of terrestrial communication that its applications cover remote regions, such as open field and desert which terrestrial bases can not support. [5] proved that, in such regions, LOS (line-of-sight) components are dominant because of the lack of shades. Therefore, LEO satellite channel is also modeled according to the conclusion.

Tapped delay line is used to model LEO satellite channel in this paper as shown in Figure 3. In formula (1), for different $\tau_{1}$ and $\tau_{2}, h\left(t, \tau_{1}\right)$ and $h\left(t, \tau_{2}\right)$ are uncorrelated. For a certain $\tau, h(t, \tau)$ is a stationary complex Gaussian stochastic process over time $t$. Therefore, formula (1) based on tapped delay line is given below

$$
h(t, \tau)=\sum_{l=0}^{L-1} b_{l} v_{l}(t) \delta\left(\tau-\tau_{l}\right)
$$

where, $\tau_{l}$ is the propagation delay of the $l$-th path; $v_{l}(t)$ is complex Gaussian process, which is the weight of the propagation delay of the $l$-th path. $b_{l}$ is the delay coefficient.

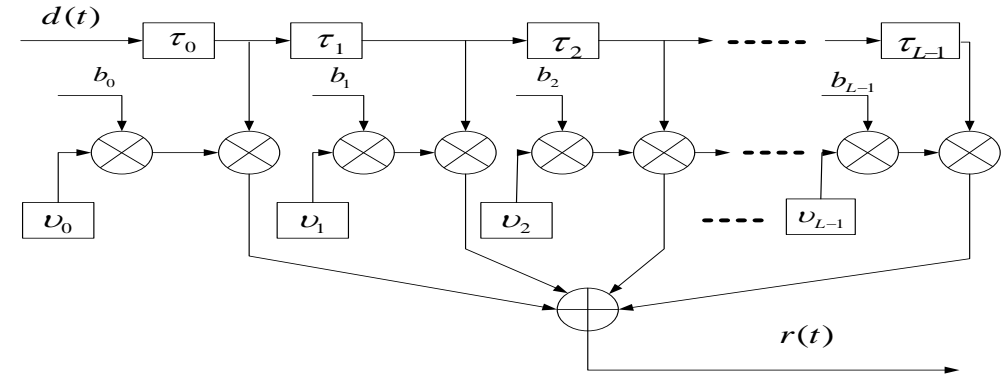

\section{Figure 3. Tapped Delay Line Model of Frequency Selective Channel for LEO Satellite System}

Let $d(t)$ and $r(t)$ stand for the input and output low-pass complex signals, respectively. With respect to the influence of channel, the output signal can be expressed as

$$
r(t)=\sum_{l=0}^{L-1} b_{l} v_{l}(t) d\left(\tau-\tau_{l}\right)
$$

In general, the amplitude $b_{0}(t)$ of LOS path is Rician distributed, while other amplitudes $b_{l}(t), l=1,2, \cdots, L-1$ of non-LOS paths are Rayleigh distributed. The simulations of Rician and Rayleigh processes need two colored complex Gaussian processes which can be implemented by superposing a series of sine waves.

In tapped delay line model for LEO satellite channel, the impulse response of multi-path channel consists of multiple paths with different delays that each path has specific amplitude fading and power spectrum. This paper uses the channel data measured by German Aerospace Center to model LEO satellite channel in the environments of urban, suburban and rural areas [6]. The carrier frequency is $1.82 \mathrm{G} \mathrm{Hz}$. Tables 1,2 and 3 present the channel parameters of above environments. 
Table 1. Channel Model Parameter in Urban Environment

\begin{tabular}{cccccc}
\hline Tap & $\begin{array}{c}\text { Distribution } \\
\text { function }\end{array}$ & Parameter & $\begin{array}{c}\text { Parameter } \\
\text { distribution }\end{array}$ & Value/dB & Delay/ns \\
\hline 1 & LOS: Rician & Rician factor & $K$ & 5.3 & 0 \\
2 & nLOS: Rayleigh & Average multi-path power & $2 \sigma_{l}^{2}$ & -12.1 & 60 \\
3 & Rayleigh & Average multi-path power & $2 \sigma_{l}^{2}$ & -17.0 & 100 \\
4 & Rayleigh & Average multi-path power & $2 \sigma_{l}^{2}$ & -18.3 & 130 \\
5 & Rayleigh & Average multi-path power & $2 \sigma_{l}^{2}$ & -22.1 & 250 \\
\hline
\end{tabular}

Table 2. Channel Model Parameter in Rural Environment

\begin{tabular}{cccccc}
\hline Tap & $\begin{array}{c}\text { Distribution } \\
\text { function }\end{array}$ & Parameter & $\begin{array}{c}\text { Parameter } \\
\text { distribution }\end{array}$ & Value/dB & Delay/ns \\
\hline 1 & LOS: Rician & Rician factor & $K$ & 6.3 & 0 \\
2 & RLOS: Rayleigh & Average multi-path power & $2 \sigma_{l}^{2}$ & -9.5 & 100 \\
3 & Rayleigh & Average multi-path power & $2 \sigma_{l}^{2}$ & -25.2 & 250 \\
\hline
\end{tabular}

Table 3. Channel Model Parameter in Suburban Environment

\begin{tabular}{cccccc}
\hline Tap & $\begin{array}{c}\text { Distribution } \\
\text { function }\end{array}$ & Parameter & $\begin{array}{c}\text { Parameter } \\
\text { distribution }\end{array}$ & Value/dB & Delay/ns \\
\hline \multirow{2}{*}{1} & LOS: Rician & Rician factor & $K$ & 9.7 & 0 \\
2 & nLOS: Rayleigh & Average multi-path power & $2 \sigma_{l}^{2}$ & -7.3 & 100 \\
3 & Rayleigh & Average multi-path power & $2 \sigma_{l}^{2}$ & -23.6 & -28.1 \\
\hline
\end{tabular}

Figure 4 - Figure 12 depict the signal amplitude and scattering function of each tap, and the scattering function of each LOS tap of tap delay line model for LEO satellite channel in the environments of urban, suburban and rural areas. 


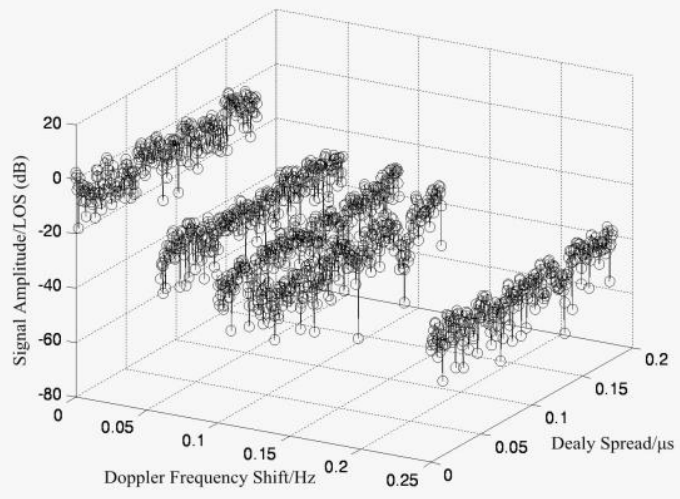

Figure 4. Signal Amplitude for each Tap (Urban Environment)

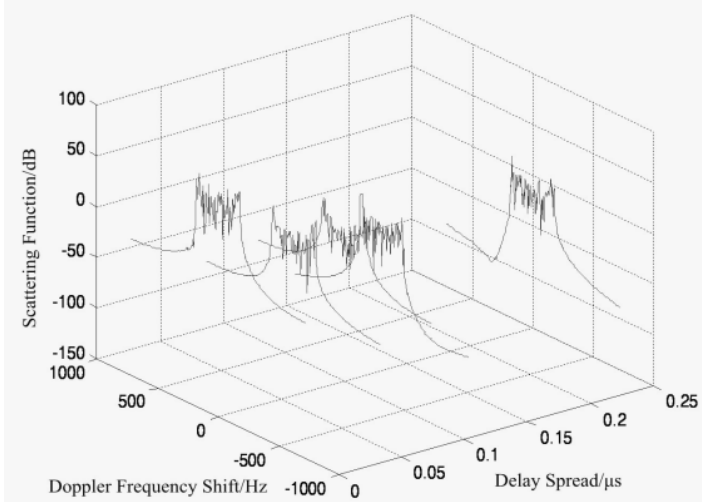

Figure 5. Scattering Function for each Tap (Urban Environment)

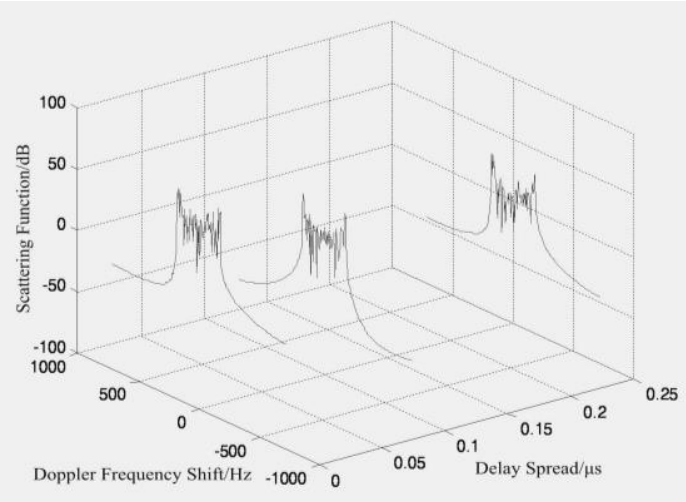

Figure 8. Scattering Function for each Tap (Rural Environment)

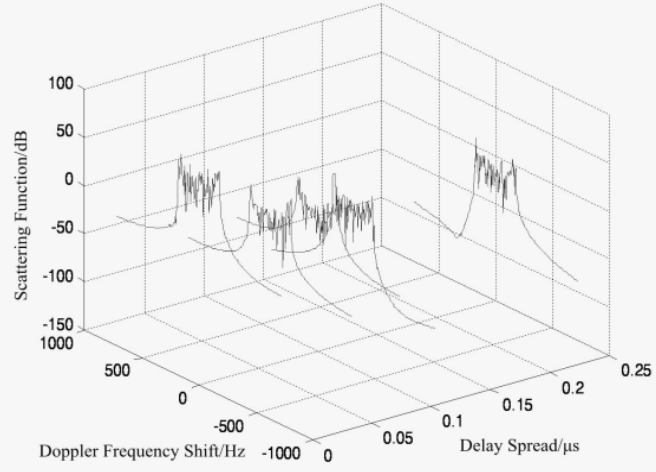

Figure 5. Scattering Function for each Tap (Urban Environment)

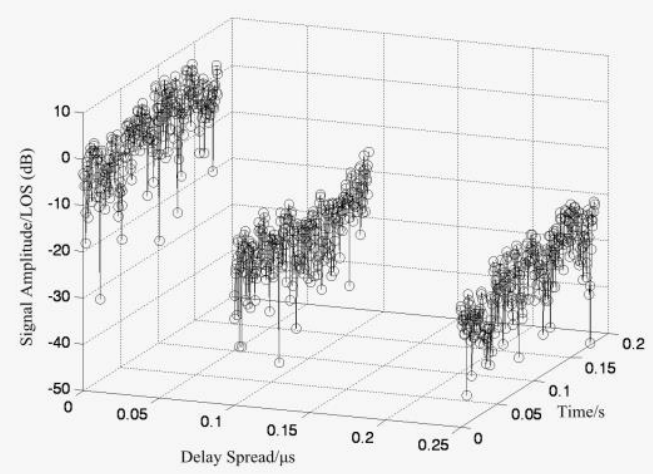

Figure 7. Signal Amplitude for each Tap (Rural Environment)

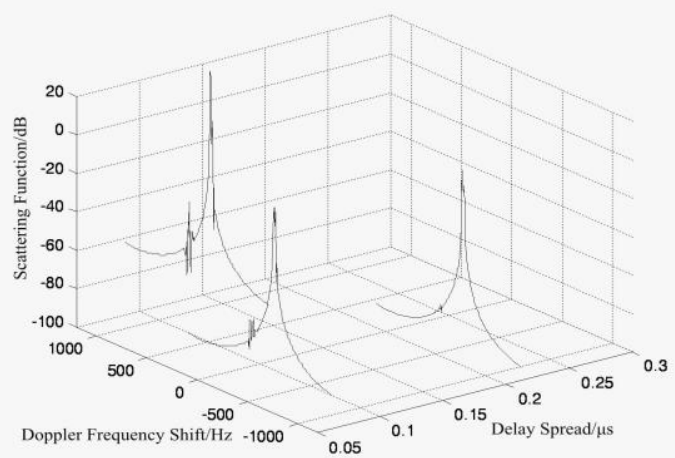

Figure 9. Scattering Function for each LOS Tap (Rural Environment) 


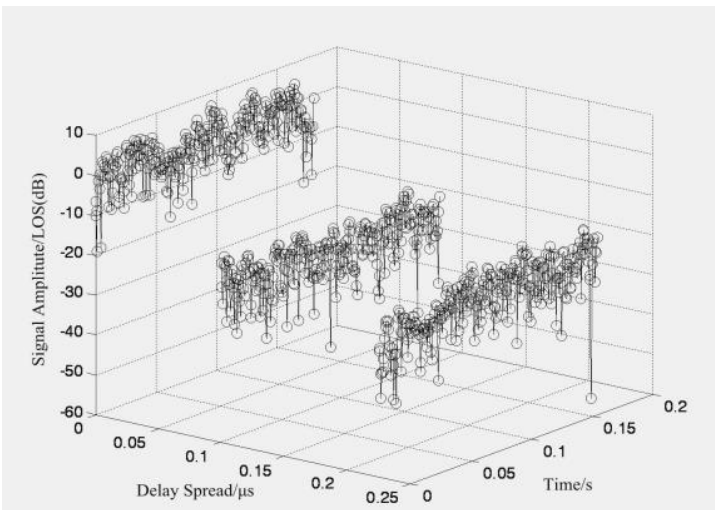

Figure 10. Signal Amplitude for each Tap (Suburban Environment)

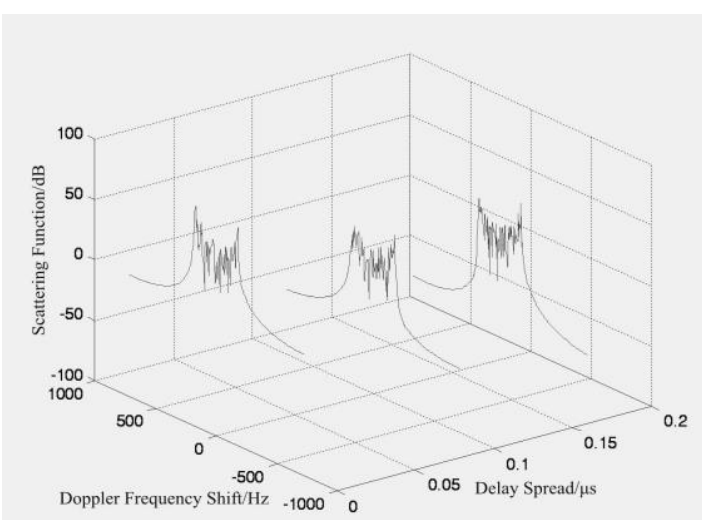

Figure 11. Scattering Function for each Tap (Suburban Environment)

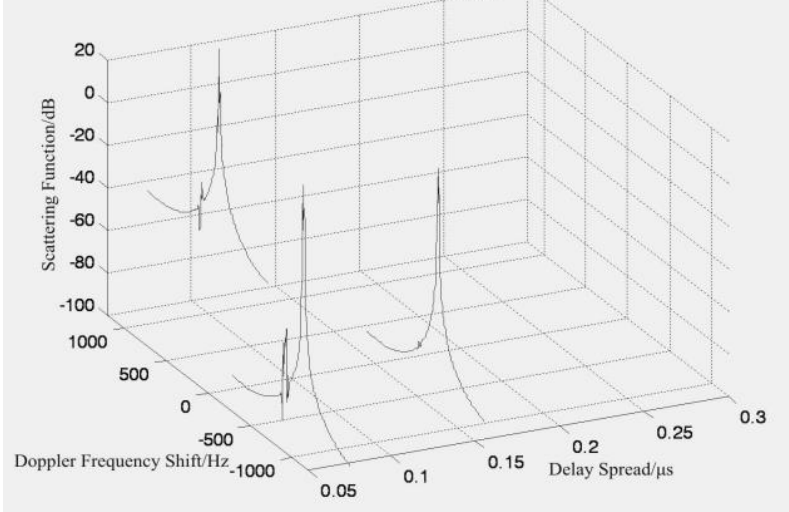

Figure 12. Scattering Function for each LOS Tap (Suburban Environment)

For different simulation scenarios, the delay and signal amplitude fading of multipaths are quite different. From above results it is obvious that the impulse response of the first path has higher amplitude mainly because there is LOS component in the first path of which envelope is Rician distributed. For urban environment, since many buildings result in signal reflection and diffraction, the received signal has larger delay spread and incurs serious fading. In contrast to urban scenario, the number of multipaths in rural areas is significantly less than the former because of few buildings in the areas, leading to flat signal fading due to the improved signal reflection and diffraction. For suburban scenario, building density is less than urban areas; in the meantime, vegetation is less than rural areas. Therefore, channel fading is flat relative to urban case because signal reflection and refraction is the weakest among three scenarios.

\section{Analysis of Throughput Performance}

Based on channel modeling, the simulation flowchart of throughput for LEO satellite system is given in Figure 13. First, the parameters for both LEO satellite system and user are initialized. With user's access, communication links are set up. According to user's CQI, 
modulation and coding modes are adaptively selected to guarantee system throughput. Specifically, if SNR (signal noise ratio) is high, high-order modulation and coding are adopted to achieve high throughput. If SNR is low, user signal may undergo deep fading, resulting in that low-order modulation and coding must be selected to guarantee the optimal trade-off between spectrum and system throughput.

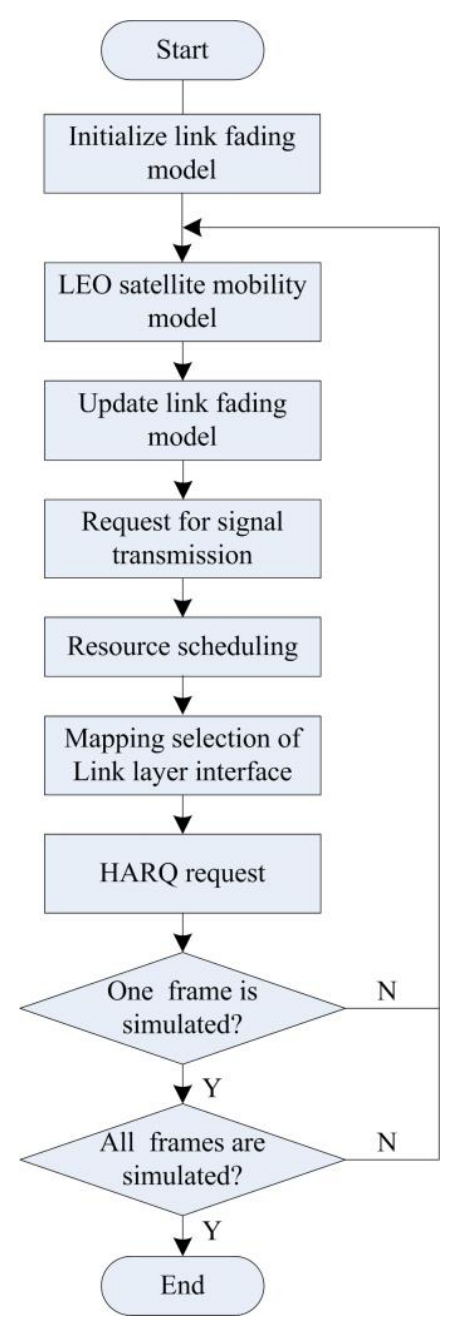

\section{Figure 13. Simulation Flowchart of Link Throughput for LEO Satellite System}

Using Monte Carlo method, all OFDM frames are simulated one by one. In Figure 4, LEO satellite position, channel fading model, HARQ and CQI feedback are the core steps. After simulating a frame, free space loss, channel fading and satellite position should be updated to simulate the next frame.

In simulations, the satellite of $770 \mathrm{~km}$ altitude is adopted as simulation model. The number of spot beam is set to 7, while its radius is $450 \mathrm{~km}$. The movement speed of LEO satellite is $7.15 \mathrm{~km} / \mathrm{s}$ (according to Globalstar system). The visible time of satellite is about 10 minutes. Carrier frequency is L-band. Simulation scenario is the rural environment analyzed in previous section. The channel model is the rural channel scenario with LOS path and 3 multipaths which had been discussed in previous section. The main path is Rician distributed with 
the maximum delay spread $250 \mathrm{~ns}$. For simplicity of analysis, this paper only takes single user throughput into account. For uplink, DFT-S OFDM is adopted, while for downlink, OFDM is used. The cyclic prefix of OFDM symbol should be set to $250 \mathrm{~ns} \times 4=1 \mu \mathrm{s}$, while the period of OFDM symbol could be set to $1 \mu \mathrm{s} \times 5=5 \mu \mathrm{s}$.

Each subframe consists of 20 OFDM symbols and each 5 subframes constitute a radio frame. Block pilot signal is used to estimate channel. Simulation step is set to one frame. The number of carriers is 1024. Single input single output (SISO) model is adopted. During each subframe, the movement of LEO satellite and beam coverage is updated, in the meantime, SNR detection, resource scheduling, CQI feedback, modulation and coding are determined as well. Every radio frame, the visible time of LEO satellite is updated. Table 4 presents the link parameters for LEO OFDM satellite system. Considering accuracy and simplicity of analysis, the adopted resource scheduling model is PF [7], while the mapping mode between physical layer and link layer is EEMS [8]. The improved RLS algorithm [9] is adopted to complete signal detection on uplink, while FSAGE algorithm [10] is adopted to detect downlink signal.

Table 4. Link Parameters for LEO OFDM Satellite System

\begin{tabular}{cc}
\hline Link parameters & Value \\
\hline EIRP on-board equipment (dBW) & 38.02 \\
Mean EIRP of mobile terminal (dBW) & -4.7 \\
Power detection threshold of receiver on board (dBW) & -156 \\
Atmospheric loss, rain attenuation (dB) & 0.7 \\
Receiver gain of terminal $(\mathrm{dB})$ & 2.6 \\
Receive antenna gain of satellite $(\mathrm{dB})$ & 3.6 \\
Bandwidth (MHz) & 10 \\
Noise temperature of on-board equipment $(\mathrm{dBK})$ & 22.8 \\
Noise temperature of of terminal (dBK) & 26.6 \\
\hline
\end{tabular}

Table 5 presents different channel characteristics for different CQI indexes according to additive white Gaussian noise (AWGN) channel, different modulation and coding modes when block error rate (BLER) is not more than 0.1. The modulation and the coding modes of $\mathrm{AMC}$ are selected according to $[11,12]$. Information transfer between physical layer and link layer is by means of CQI, while the values of CQI are determined by the modulation and the coding modes of physical layer.

Table 5. CQI Correspondence to Physical Modulation and Code Pattern

\begin{tabular}{ccccc}
\hline CQI index & Modulation mode & Code rate & Mapping coefficient & SNR threshold \\
\hline 1 & QPSK & 0.0762 & 1.71 & -5.50 \\
2 & QPSK & 0.1172 & 1.32 & -3.75 \\
3 & QPSK & 0.1185 & 1.35 & -2.75 \\
4 & QPSK & 0.3008 & 1.78 & -0.75 \\
5 & QPSK & 0.4385 & 1.77 & 0.75 \\
6 & QPSK & 0.5879 & 1.45 & 2.50 \\
7 & 16QAM & 0.3691 & 4.52 & 6.0
\end{tabular}


International Journal of Future Generation Communication and Networking Vol.6, No.6 (2013)

\begin{tabular}{ccccc}
8 & 16QAM & 0.4785 & 5.22 & 6.5 \\
9 & 16QAM & 0.6016 & 4.57 & 8.75 \\
10 & 64QAM & 0.4551 & 4.13 & 13 \\
11 & 64QAM & 0.5537 & 5.07 & 14 \\
12 & 64QAM & 0.6504 & 4.94 & 14.75 \\
13 & 64QAM & 0.7539 & 8.39 & 16 \\
14 & 64QAM & 0.8525 & 15.22 & 18 \\
15 & 64QAM & 0.9258 & 20.91 & 19.25 \\
\hline
\end{tabular}

Figure 14 presents the relationship between CQI and the received SNR. It demonstrates that user terminal can transmit information through selecting adequate mapping and coding mode according to CQI determined by SNR. Figure 15 presents the BLER performance for different CQI values under AWGN.

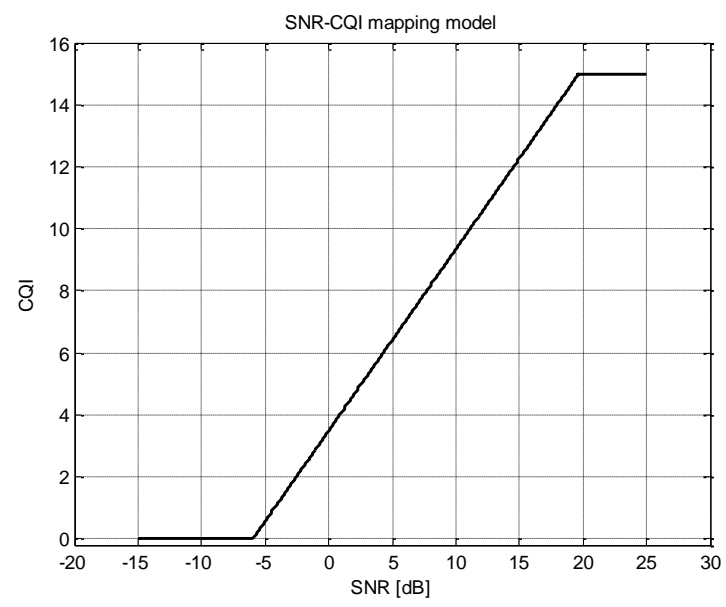

Figure 14. Mapping for SNR vs. CQI

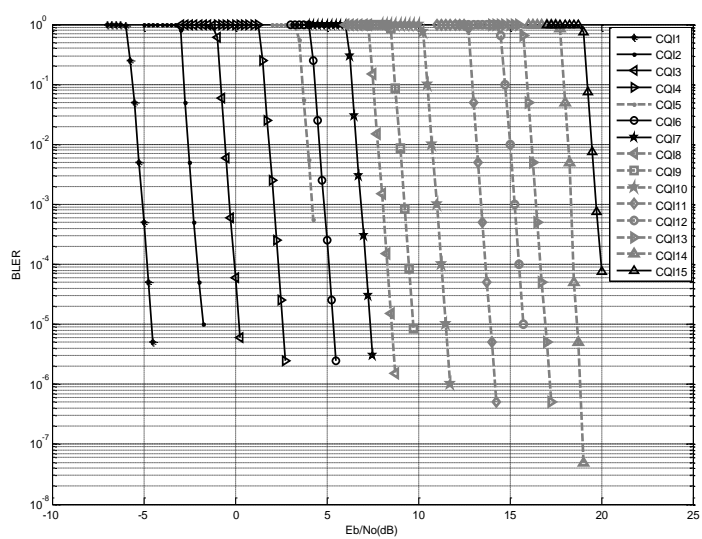

Figure 15. BLER Performance of Different CQI (with bandwidth 10MHz) 
Figure 16 demonstrates the relationship between throughput and different CQI values under the conditions of $10 \mathrm{MHz}$ bandwidth and AWGN. It is obvious that with the increase of CQI, system throughput increases under high SNR mainly because high-order modulation is adopted.

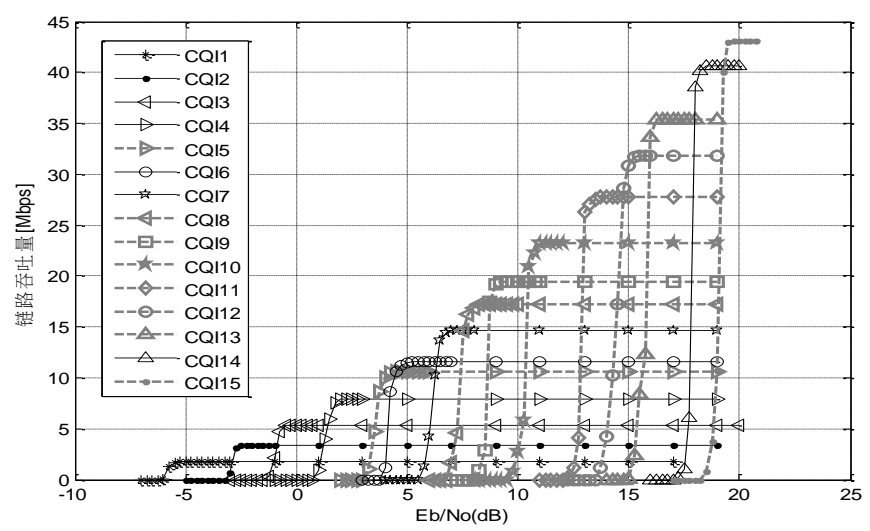

Figure 16. Throughput Performance of Different CQI in AWGN (with Bandwidth 10MHz)

Figure 17 shows the throughput performance under the conditions of $10 \mathrm{MHz}$ bandwidth and AWGN when AMC is adopted. It is apparent that when SNR is low, low-order mapping and coding mode is adopted to guarantee reliable signal transmission. When SNR is high, high-order mapping and coding mode is adopted to maximize system throughput.

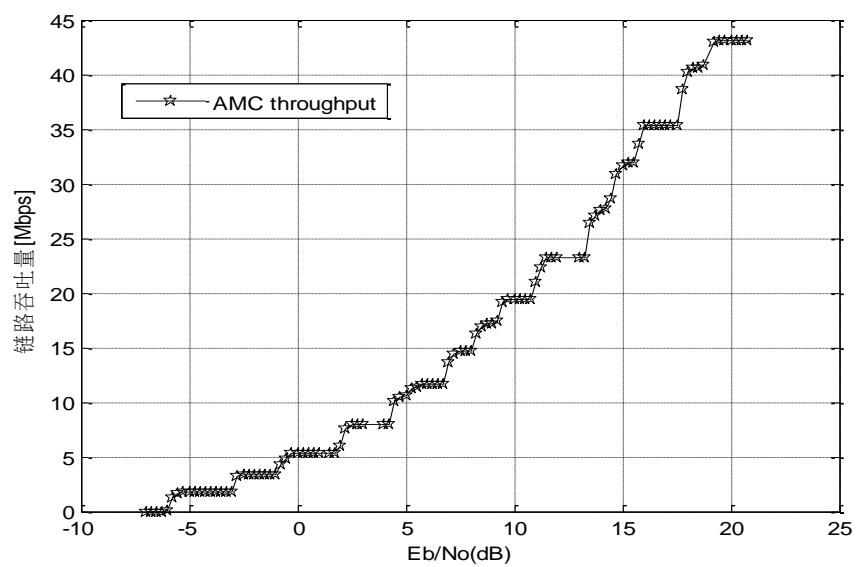

Figure 17. Throughput Performance of AMC in AWGN (with Bandwidth 10MHz)

Figure 18 gives the relationship between throughput and different CQI values for rural scenario under the conditions of $10 \mathrm{MHz}$ bandwidth. Compared with AWGN channel conditions, system throughput decreases mainly because multi-path fading. 


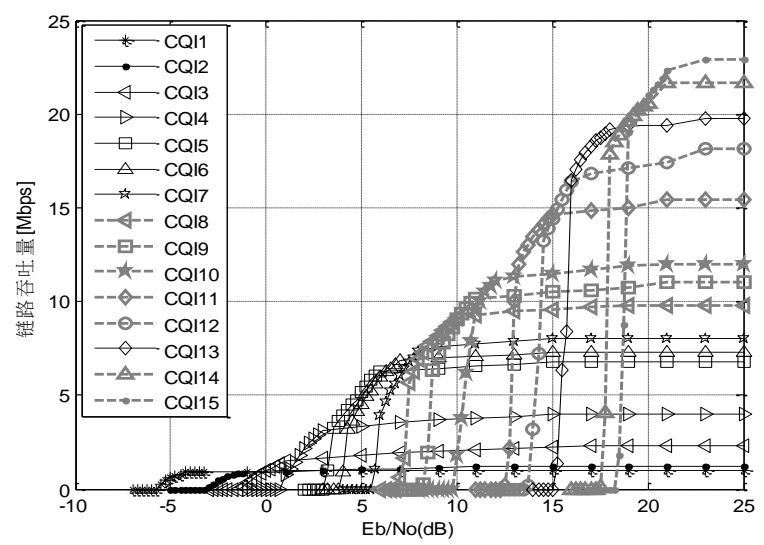

\section{Figure 18. Throughput Performance of CQI in Rural Environment Channel Model (with Bandwidth 10MHz)}

Finally, the throughput of LEO OFDM satellite system is compared with the throughput of LEO MF-TDMA satellite system. MF-TDMA is the combination of FDMA (Frequency Division Multiple Access) and TDMA (Time Division Multiple Access). As the popular transmission scheme for broadband satellite communication system, MF-TDMA can support user terminals sharing a series of carriers of different rates, while several time slots share the same carrier. Therefore, resource is flexibly allocated in both time and frequency domains. In our simulation, 20 TDMA symbols constitute a subframe, while 5 subframes constitute a MFTDMA super frame. The number of time slot for a carrier is 256 and each time slot is uniformly distributed in a subframe. The receiver on board completes signal detection according to MMSE (Minimum Mean Square Error) criterion. Figure 19 presents the throughput comparison of OFDM and MF-TDMA transmission schemes for rural scenario under the conditions of $10 \mathrm{MHz}$ bandwidth. Compared with MF-TDMA scheme, as OFDM adopts orthogonal subcarriers, the latter has better anti-fading effect. It is beneficial to higher link throughput.

\section{Conclusions}

This paper analyzes the throughput with AMC for LEO OFDM satellite communication system. Specifically, throughput analysis flow for LEO OFDM system is presented, and then channel model for LEO satellite communication system is expounded. Further, beam coverage and signaling models are introduced. Considering the fast movement of LEO satellite and channel fading, user terminal should update CQI and select adequate modulation and coding mode. By comparing the throughput performances of LEO OFDM communication system with LEO MF-TDMA communication system, it is shown that the former outperforms the latter in system throughput.

\section{Acknowledgements}

This paper is supported by National Natural Science Foundation of China under grant No. 61301131 and the Fundamental Research Funds for the Central Universities under grant No. 3132014210 and 3132013337-3-2. The Communication Research Center of Harbin Institute of Technology gives some constructive suggestions to the algorithm designs and simulations. 


\section{References}

[1] Z. Y. Na, "Research on Qos Guarantee Methods in Satellite Internet", Dissertation of Harbin Institute of Technology, Harbin, China, (2010).

[2] C. M. Chen and W. J. Zeng, "The Adaption of LTE Interface in Satellite Communication", Wireless Communication Technology, vol. 4, (2011).

[3] X. Zhang, "Research and System-level simulation Verification of key technologies for 3GPP LTE", Dissertation of Wuhan University of Technology, Wuhan, China, (2008).

[4] C. Sun, "Research on System-Level Simulation Platform Implementation for LTE-Advanced Downlink and its Key Technologies", Dissertation of Beijing University of Posts and Telecommunications, Beijing, China, (2009).

[5] W. J. Vogel and J. Goldhirsh, "Fade Measurements at L-band and UHF in Mountainous Terrain for Land Mobile Satellite Systems", IEEE Transactions on Antennas and Propagation, vol. 36, no. 1, (1988).

[6] E. Lutz, M. Werner and A. Jahn, "Satellite Systems for Personal and Broadband Communication", Sprionger-Verlag, Berlin Heideberg, (2000).

[7] W. Chen, Y. Sun, Y. Z. Li and C. Luo, "Realization of System-Level Simulation Platform of LTE Based on MATLAB", Communications Technology, vol. 43, no. 5, (2010).

[8] H. M. Wang and T. Esa, "Effective SINR Approach of Link to System Mapping in OFDM/Multi-Carrier Mobile Network", Telecommunication Engineering, vol. 1, (2006).

[9] Q. Y. Guan, H. L. Zhao and Q. Guo, "Elimination of Carrier Frequency Offset in Uplink OFDMA System Based on TD-LMS Algorithm", Journal of South China University of Technology (Natural Science Edition), vol. 38, no. 5, (2010).

[10] Q. Y. Guan, "Research on OFDM Transmission Technique for Broadband LEO Satellite Communication System", Dissertation of Harbin Institute of Technology, Harbin, China, (2011).

[11] 3rd Generation Partnership Project, "Physical layer procedures for evolved universal terrestrial radio access (E-UTRA)", 3GPP TS 36.213, 3GPP, (2009).

[12] P. Christos, D. Nikos and T. Leandros, "Dynamic Radio Resource and Interference Management for MIMOOFDMA Mobile Broadband Wireless Access Systems", Computer Networks, vol. 57, no. 1, (2013).

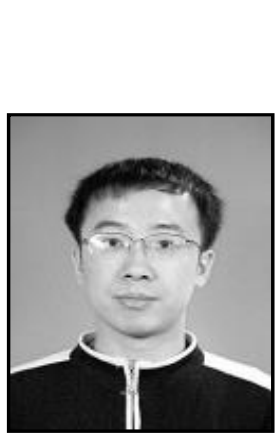

\section{Author}

Zhenyu Na, was born in 1981, Harbin, P. R.China. He received the B.S. degree in communication engineering, M.S. degree in information and communication engineering from Harbin Institute of Technology (HIT) in 2004 and 2007, respectively. In Jan. 2010, he received doctoral degree in information and communication engineering at Communication Now, he is a lecturer at School of Information Science and Technology of Dalian Maritime University (DMU) with research interests including satellite communications and networks, wireless networks, MIMOOFDM communications. Until now, he has published 15 papers as the first author in international journals and conferences. 
International Journal of Future Generation Communication and Networking Vol.6, No.6 (2013) 